\title{
Adequate nephroprotection reduces renal complications after hyperthermic intrathoracic chemotherapy
}

\author{
Till Markowiak $^{1} \odot \mid$ Nadine Kerner ${ }^{1}$ | Reiner Neu MD ${ }^{1}$ | Tobias Potzger MD ${ }^{1} \mid$ \\ Christian Großer $^{2}$ | Florian Zeman ${ }^{3}$ | Hans-Stefan Hofmann MD ${ }^{1,2}$ | Michael Ried MD ${ }^{1}$
}

${ }^{1}$ Department of Thoracic Surgery, University Medical Center Regensburg, Regensburg, Germany

${ }^{2}$ Department of Thoracic Surgery, Hospital Barmherzige Brüder Regensburg, Regensburg, Germany

${ }^{3}$ Center for Clinical Studies, University Medical Center Regensburg, Regensburg, Germany

\section{Correspondence}

Michael Ried, MD, Department of Thoracic Surgery, University Medical Center Regensburg, Franz-Josef-Strauß-Allee 11, D-93053 Regensburg, Germany.

Email: michael.ried@ukr.de

\begin{abstract}
Background and Objectives: Hyperthermic intrathoracic chemotherapy (HITOC) is used for the treatment of malignant pleural tumors. Although HITOC proved to be safe, postoperative renal failure due to nephrotoxicity of intrapleural cisplatin
\end{abstract} remains a concern.

Methods: This single-center study was performed retrospectively in patients who underwent pleural tumor resection and HITOC between September 2008 and December 2018.

Results: A total of 84 patients (female $n=33$; 39.3\%) with malignant pleural tumors underwent surgical cytoreduction with subsequent HITOC $\left(60\right.$ minutes; $\left.42^{\circ} \mathrm{C}\right)$. During the study period, we gradually increased the dosage of cisplatin $\left(100-150 \mathrm{mg} / \mathrm{m}^{2} \mathrm{BSA} n=36\right.$; $175 \mathrm{mg} / \mathrm{m}^{2} \mathrm{BSA} \mathrm{n}=2$ ) and finally added doxorubicin (cisplatin $175 \mathrm{mg} / \mathrm{m}^{2} \mathrm{BSA} /$ doxorubicin $65 \mathrm{mg} ; \mathrm{n}=46)$. All patients had perioperative fluid balancing. The last $54(64.3 \%)$ patients also received perioperative cytoprotection. Overall 29 patients (34.5\%) experienced renal insufficiency. Despite higher cisplatin concentrations, patients with cytoprotection showed significantly lower postoperative serum creatinine levels after 1 week $(P=.006)$ and at discharge $(P=.020)$. Also, they showed less intermediate and severe renal insufficiencies (5.6\% vs $13.3 \%)$.

Conclusions: Adequate perioperative fluid management and cytoprotection seem to be effective in protecting renal function. This allows the administration of higher intracavitary cisplatin doses without raising the rate of renal insufficiencies.

\section{KEYWORDS}

cytoprotection, hyperthermic intrathoracic chemotherapy, hyperthermic intrathoracic chemotherapy, nephroprotection, renal insufficiency

\section{1 | INTRODUCTION}

Hyperthermic intrathoracic chemotherapy (HITOC) offers an additional treatment option for malignant pleural tumors after surgical cytoreduction. Especially it is used to further improve local tumor control in malignant pleural mesothelioma (MPM) and thymic malignancies with pleural spread, who underwent multimodality therapy including surgical resection. ${ }^{1}$ Some previous studies showed promising results with regard to recurrence-free survival and longterm. ${ }^{2}$ Furthermore, HITOC proved to be safe with tolerably low rates of complications. ${ }^{2-4}$ Nevertheless, certain aspects in the perioperative management of HITOC require special attention. Particularly known is the dose-dependent renal toxicity of cisplatin, which is an integral part of the perfusion solution. ${ }^{5}$ Moderate to 
severe nephrotoxicity was noted in $25 \%$ to $33 \%$ of patients receiving a single intravenous dose of cisplatin of 50 to $75 \mathrm{mg} / \mathrm{m}^{2}$ body surface area (BSA). ${ }^{6}$ Studies focusing on HITOC reported rates of postoperative renal dysfunction from $3.3 \%$ to $57 \%{ }^{3,7,8}$

To avoid this serious complication, adequate hydration of the patient (and forced diuresis if necessary) is generally recommended. ${ }^{9}$ In addition to this perioperative fluid management, however, drug therapy for renal cytoprotection is also available. In the recent literature, this cytoprotection most commonly consists of a combination of amifostine and sodium thiosulfate. ${ }^{2,3,10}$ The pretreatment with amifostine is intended to reduce tubulotoxicity and sodium thiosulfate protects against renal damage by inactivating cisplatin through covalent binding. ${ }^{5,11}$

There are only very few studies investigating the influence of this nephroprotective regime on postoperative renal function in patients undergoing HITOC. Therefore, the objective of this study was to investigate the rate of renal insufficiency and the postoperative course of serum creatinine after HITOC depending on the cisplatin concentration and the administration of cytoprotective agents.

\section{2 | MATERIALS AND METHODS}

\section{1 | Study design}

This retrospective, single-center study included all patients who received pleural tumor resection followed by HITOC from September 2008 to December 2018 ( $n=84$ ). Approval by our institutional ethics committee was given before data collection and informed consent was obtained from all patients. During the hospital stay data concerning the performed surgery and subsequent HITOC were enrolled within the patient records.

The minimum preoperative threshold of glomerular filtration rate (GFR) was more than $60 \mathrm{~mL} /$ minute $/ 1.73 \mathrm{~m}^{2}$. Serum creatinine levels were routinely collected (preoperative, postoperative, first and second postoperative day, after 1 week and at the time of discharge) to assess renal function after major surgery. Based on risk of renal failure and acute kidney injury network classification, renal insufficiency grade 1 was defined as an increase in serum creatinine more than $0.3 \mathrm{mg} / \mathrm{dL}$ or doubling over 48 hours, a two to three-fold increase as stage 2 and a stage 3 consisted of a three-fold increase or serum creatinine more than $4 \mathrm{mg} / \mathrm{dL}^{12,13}$ Also the need for postoperative dialysis was registered.

The primary endpoint of the study was to determine postoperative renal insufficiency after surgical cytoreduction including HITOC. Secondary endpoints included the impact of the applied therapy (cisplatin dosage, application of cytoprotection, and surgical procedure) on postoperative serum creatinine levels.

\section{2 | Surgical resection and HITOC}

Depending on the location of the tumor, pleurectomy/decortication $(P / D)$, extended $P / D(e P / D)$ including resection of the pericardium and/or diaphragm and/or lung resection or extrapleural pneumonectomy (EPP) was carried out. A macroscopic complete resection and HITOC in one session was always aimed at. As perfusion system, the ThermoChemo HT1000 was used. Three to four liters of sodium chloride served as priming-volume until a stable circulation could be established. The dosage of the subsequently added chemotherapeutic agent was stepwise increased throughout the study. In the beginning, a dose of 100 to $150 \mathrm{mg} / \mathrm{m}^{2}$ BSA was used. Later, cisplatin was increased to $175 \mathrm{mg} / \mathrm{m}^{2}$ BSA and combined with $65 \mathrm{mg}$ doxorubicin. About this time, additional cytoprotection was also introduced. If there were contraindications for the use of the maximum cisplatin dose (eg, reduced renal function), a lower dose was used. The perfusion was performed for 60 minutes at $42^{\circ} \mathrm{C}$ with a flow of approximately $1.5 \mathrm{~L} /$ minute.

\section{3 | Nephroprotection}

All patients received pretreatment hydration by application of approximately $2 \mathrm{~L}$ of oral fluid on the day before surgery. If the patient did not reach this amount, we administered an additional one liter of fluid intravenously. In the perioperative period, a liberal fluid regime was obtained in all patients to keep the diuresis more than $100 \mathrm{~mL} /$ hour during surgery and for the first postoperative days. Starting in June 2013, we complemented this regime by using additional cytoprotection as described by Sugarbaker et al., ${ }^{5,10}$ The intraoperative cytoprotection consisted of intravenous amifostine and sodium thiosulfate. Amifostine was given in a dosage of $910 \mathrm{mg} / \mathrm{m}^{2}$ BSA 30 minutes before the initiation of the HITOC. Sodium thiosulfate was administered directly afterward $\left(4 \mathrm{~g} / \mathrm{m}^{2}\right.$ BSA) and continuously over a period of 6 hours ( $\left.12 \mathrm{~g} / \mathrm{m}^{2} \mathrm{BSA}\right)$ after the HITOC at the intensive care unit.

\section{4 | Statistical analysis}

Data collection and statistical analyses were performed using IBM SPSS Statistics, Version 24 (IBM Corporation, Armonk, NY). Categorical data were summarized as absolute numbers and percentages and were compared between groups using the $\chi^{2}$ test of independence or Fisher's exact test. Interval scaled data were presented as mean \pm standard deviation or as median (IQR) and were compared using Student's t-test or the Mann-Whitney U-test depending on the underlying distribution of the variable. The distribution was analyzed using the Shapiro-Wilk test. To examine the influence of cytoprotective therapy on postoperative creatinine levels, an analysis of covariance was performed with preoperative creatinine levels set as a covariate. $P<.05$ was considered statistically significant for all analyses. A logistic regression model was performed to assess the relationship between predictor variables to a binary response variable.

\section{3 | RESULTS}

\section{1 | Demographic data}

The median age of the study population was 57 (IQR 16) years, and $33(39.3 \%)$ of patients were female (Table 1$)$. Most patients $(n=43$, 
TABLE 1 Characteristics of the study population

\begin{tabular}{|c|c|c|c|}
\hline & Study sample $(n=84)$ & Cytoprotective treatment $(n=54)$ & Hydration only $(n=30)$ \\
\hline Female sex, $\mathrm{n}(\%)$ & $33(39.3)$ & $24(44.4)$ & $9(30)$ \\
\hline Median age; IQR, y & $57 ; 16$ & $57 ; 16$ & $57 ; 22$ \\
\hline Median preoperative GFR; IQR, $\mathrm{mL} / \mathrm{min} / 1.73 \mathrm{~m}^{2}$ & $96.5 ; 23.2$ & $96.7 ; 23$ & $94.2 ; 27.8$ \\
\hline Induction therapy; n (\%) & $53(63.1)$ & $27(50)$ & $26(86.7)$ \\
\hline $\begin{array}{l}\text { Procedure; } n \text { (\%): } \\
\text { P/D } \\
\text { eP/D } \\
\text { EPP }\end{array}$ & $\begin{array}{l}30(35.7) \\
46(54.8) \\
7(8.3)\end{array}$ & $\begin{array}{l}14(25.9) \\
36(66.6) \\
3(5.5)\end{array}$ & $\begin{array}{l}16(53.3) \\
10(33.3) \\
4(13.3)\end{array}$ \\
\hline Resection + HITOC in one session, $n(\%)$ & $82(97.6)$ & $53(98.1)$ & $29(96.7)$ \\
\hline $\begin{array}{l}\text { Cisplatin dosage, } \mathrm{n}(\%) \\
\text { Cisplatin } 100-150 \mathrm{mg} / \mathrm{m}^{2} \\
\text { Cisplatin } 175 \mathrm{mg} / \mathrm{m}^{2} \\
\text { Cisplatin } 175 \mathrm{mg} / \mathrm{m}^{2}+\text { doxorubicin } 65 \mathrm{mg}\end{array}$ & $\begin{array}{l}36(42.9) \\
2(2.4) \\
46(54.8)\end{array}$ & $\begin{array}{l}6(11.1) \\
2(3.7) \\
46(85.2)\end{array}$ & $\begin{array}{l}30(100) \\
0(0) \\
0(0)\end{array}$ \\
\hline Mean inflow volume $\pm S D, m L$ & $4202 \pm 929$ & $4243 \pm 965$ & $4130 \pm 871$ \\
\hline Mean duration of operation $\pm S D$, min & $300 \pm 90$ & $315 \pm 69$ & $275 \pm 92.7$ \\
\hline Median ICU-stay; IQR, d & $2 ; 3$ & $3 ; 4$ & $1 ; 1.5$ \\
\hline
\end{tabular}

Abbreviations: BSA, body surface area; eP/D, extended P/D; EPP, extrapleural pneumonectomy, GFR, glomerular filtration rate; HITOC, hyperthermic intrathoracic chemotherapy; P/D, pleurectomy/decortication; ICU, intensive care unit; IQR, interquartile range; MPM, malignant pleural mesothelioma; SD, standard deviation.

$51.2 \%)$ suffered from MPM or thymoma with pleural spread (stage IVa: $n=32,38.1 \%$ ). Considerably fewer patients showed secondary pleural carcinosis ( $n=9,10.7 \%$ ) of extrathoracic tumors. With $63.1 \%$ ( $n=53$ ), more than half of the patients received an induction therapy before surgery and HITOC. The preoperative GFR in the median was $96.5 \mathrm{~mL} /$ minute $/ 1.73 \mathrm{~m}^{2}$ (IQR 23.2) and ranged from 60.5 to $147.4 \mathrm{~mL} /$ minute $/ 1.73 \mathrm{~m}^{2}$.

\section{2 | Surgical resection and HITOC}

The most frequently performed surgery was eP/D in 46 patients (54.8\%; Table 1). In only seven patients (8.3\%) EPP was necessary. It was feasible to carry out surgical resection and HITOC in the same session in almost all cases $(n=82 ; 97.6 \%)$. In two patients a second session for HITOC was necessary, one and 19 days later due to an extended tumor resection with hemodynamic instability. Concerning the chemotherapeutic drugs used in HITOC, most patients $(n=48$; $57.1 \%$ ) were treated with cisplatin at a dosage of $175 \mathrm{mg} / \mathrm{m}^{2} \mathrm{BSA}$, which was additionally combined with doxorubicin $65 \mathrm{mg}$, except in two cases. In 36 patients (42.9\%), cisplatin was used alone in a dosage ranging between 100 to $150 \mathrm{mg} / \mathrm{m}^{2}$ BSA. The mean inflow volume was $4202 \pm 929 \mathrm{~mL}$ (P/D +eP/D: $4195 \pm 908 \mathrm{~mL}$; $E P P$ : $4286 \pm 1220 \mathrm{~m}$ ). The average operation time including HITOC was $300 \pm 90$ minutes. Cytoprotective therapy was applied to 54 patients (64.3\%).

\section{3 | Renal insufficiency}

In the postoperative period, overall 29 patients (34.5\%) experienced renal insufficiency grades 1 to 3 . Most of these patients $(n=22$, $75.9 \%)$ experienced only a slight elevation in creatinine $(>0.3 \mathrm{mg} / \mathrm{dL}$ or 1.5-2-fold increase) and were therefore classified as renal insufficiency grade 1. Renal dysfunctions grade 2 (2-3-fold increase; $n=4,13.8 \%$ ) and grade 3 (>three-fold increase or serum creatinine $>4 \mathrm{mg} / \mathrm{dL} ; \mathrm{n}=3,10.3 \%$ ) occurred less frequently (Table 2 ).

The calculated intrathoracic cisplatin concentration (dosage $x$ $\mathrm{BSA}$ /inflow volume) was significantly higher in patients receiving cisplatin in a dose of $175 \mathrm{mg} / \mathrm{m}^{2}$ BSA $(P=.049)$ and patients with cytoprotection $(P=.016)$ (Table 2$)$. There were no significant differences in the occurrence of renal insufficiency with regard to 
TABLE 2 Postoperative renal insufficiency

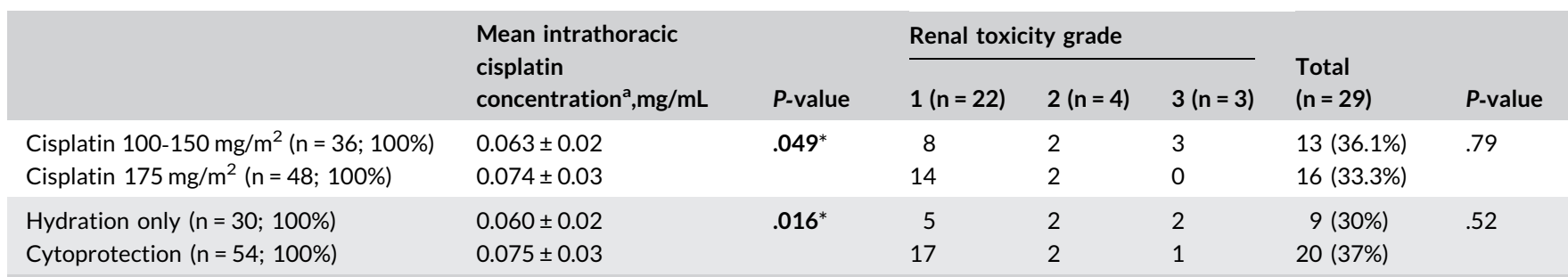

Abbreviation: BSA, body surface area.

" $P<.05$.

${ }^{\mathrm{a} C}$ Calculation of intrathoracic cisplatin concentration: (dosage $\times \mathrm{BSA} /$ inflow volume).

the dosage of cisplatin $(P=.79)$ or the application of cytoprotection $(P=.52)$. In the patients with only hydration (2008-2013), 20 cases $(37 \%)$ of renal insufficiency were recorded. In patients who additionally received amifostine and sodium thiosulfate (20132018), a total of nine patients (30\%) were affected, although these patients received higher dosages of cisplatin. Temporary dialysis was necessary in three cases (3.6\%), two of them were early patients without cytoprotective treatment. The patient requiring dialysis who received cytoprotective treatment showed normal creatinine levels 1 week after HITOC, but later developed a septic shock with renal failure making dialysis necessary. No chronic dialysis was needed.

\section{4 | Postoperative creatinine levels}

To assess renal function, serum creatinine levels were examined as renal function markers in the postoperative period depending on the applied therapy (Table 3). Patients receiving cisplatin $175 \mathrm{mg} / \mathrm{m}^{2}$ BSA showed significantly lower levels after 1 week $(P=.001)$ and at discharge $(P=.001)$ than patients receiving lower doses of cisplatin (Figure 1). Concerning cytoprotection, significant lower creatinine levels could also be observed after 1 week $(P=.006)$ as well as at discharge $(P=.020)$ (Figure 2$)$. In the same way, creatinine levels were significantly increased after EPP compared to lung-sparing P/D or $\mathrm{eP} / \mathrm{D}$.

In a logistic regression model the influence of cytoprotection, cisplatin dosage, performed surgery (P/D and eP/D vs EPP), and induction therapy on the development of postoperative renal insufficiency (grade 1-3) was investigated. A significant effect of cytoprotection $(P=.043)$ and induction therapy (0.033) was found. Regarding the dosage of cisplatin $(P=.131)$ and performed surgery $(P=.52)$, in contrast, no significant correlation was observed.

\section{4 | DISCUSSION}

HITOC offers the advantage of direct contact of the chemotherapeutics to residual tumor cells that remained in the pleural cavity after surgical cytoreduction. ${ }^{1}$ The application of high cisplatin dosages in the perfusate lead to good local concentrations in the uppermost layers of the tissue (approximately 3-4 mm), while the resulting systemic exposure amounts to only a very low fraction of the intrathoracic concentration. ${ }^{14-16}$ The existing studies already demonstrated reasonable low rates of HITOC-related complications in the perioperative period. ${ }^{2,3,10}$ However, especially postoperative renal insufficiency remains a clinically relevant concern. Reported rates of renal dysfunction after HITOC differ from $3.3 \%$ to $57 \%$, which may already indicate that relevantly different strategies are used to prevent this complication., 3,7

Especially high cisplatin doses are associated with postoperative renal complications. ${ }^{5}$ In cisplatin-based chemotherapy regimens, hydration is generally introduced as a nephroprotective strategy. ${ }^{9}$ Since excessive volume infusion can contribute to respiratory failure especially in patients undergoing lung surgery, additional diuresis, and fluid balance may be required. ${ }^{17}$ In a rat model investigating forced diuresis after cisplatin, the creatinine profiles of the forced diuresis-treated rats were similar to those of the control rats. The onset of nephrotoxicity was observed on days 2 to 3 and maximum creatinine was reached around days 4 to 5 in all rats, suggesting that forced diuresis treatment might have no impact on the underlying signaling pathways but rather attenuates the intensity of toxic signals. ${ }^{18}$

In our study, there was no significant difference in preoperative creatinine levels with regard to previous induction therapy $(P=.1)$. Postoperative renal insufficiency occurred in $34.5 \%$ of patients in our study. Sugarbaker et $\mathrm{al}^{2}$ reported $76 \%$ of patients with creatinine elevation (10\% requiring dialysis) using amifostine and $57 \%$ patients with renal toxicity using thiosulfate after HITOC. ${ }^{5,7}$ In comparison, the occurrence of postoperative renal complications using both agents appeared low. Most renal insufficiencies (75.9\%) have only become apparent through a mild rise in the serum creatinine (grade 1 ) in our patients, which was clinically not relevant. Renal dysfunction grade $2(n=4)$ and grade $3(n=3)$ occurred less frequently. Even though doxorubicin was added to the perfusate and the dosage of cisplatin was gradually increased to a dosage of $175 \mathrm{mg} / \mathrm{m}^{2}$ BSA, no significantly higher rate of renal insufficiencies occurred $(P=.79)$. The missing increase in renal complications despite higher intracavitary concentrations of cisplatin and the additive use of doxorubicin is most likely a result of the additional introduction of cytoprotection. A positive effect of cytoprotection on the rate of postoperative renal insufficiencies was also found in a multivariate analysis $(P=.043)$. The increase in grade 1 renal dysfunctions can 
be attributed to the concurrent introduction of higher cisplatin doses and cytoprotection. Moderate and severe renal dysfunction (grade 2 and 3) was more common in patients without cytoprotection (13.3\%) than in patients with cytoprotection (5.6\%), even though nearly all of them received cisplatin at the highest dose of $175 \mathrm{mg} / \mathrm{m}^{2}$ in this study. The maximum-tolerated dose of cisplatin for HITOC is suggested at $225 \mathrm{mg} / \mathrm{m}^{2}$ BSA, because higher concentrations showed significantly more renal complications, although survival rates were better., ${ }^{5,7}$ In the current literature, cisplatin is mostly administered between 150 to $175 \mathrm{mg} / \mathrm{m}^{2}$ BSA. ${ }^{10,14}$ The existing studies mostly focused on mesothelioma patients. We increased the dosage of cisplatin during the study as higher doses demonstrated a better survival in patients with MPM in the literature. ${ }^{5,7}$ We expected that also in patients with thymoma an increased dosage would lead to better long-term results.

Amifostine is preferentially absorbed by healthy cells by a factor of 100 compared to tumor cells. As the therapeutic benefit of chemotherapy is not attenuated by amifostine, administration before cisplatin is recommended for optimal distribution., ${ }^{5,21}$ WeichertJacobsen et al $^{11}$ showed that treatment with amifostine before cisplatin administration resulted in a slight urinary $N$-acetyl- $\beta$-Dglucosaminidase (NAG) leakage (day 2, $2.8 \pm 1.8$ units/gram creatinine; day 4, $13.8 \pm 13$ units/gram creatinine; normalization at day 6) compared to no cytoprotection.

In contrast to amifostine, the mechanisms of sodium thiosulfate involve inactivation of cisplatin due to covalent binding at the expense of the therapeutic benefit. Therefore, sodium thiosulfate rescue is applied after the completion of intracavitary perfusion. ${ }^{22,23}$ In the setting of high-dose intracavitary lavages (cisplatin $225 \mathrm{mg} / \mathrm{m}^{2}$ BSA) the combination of both cytoprotective drugs showed an advantage over monotherapy with sodium thiosulfate in terms of postoperative creatinine increase. ${ }^{24}$ In another study with nearly 40.000 patients, Kork et $\mathrm{al}^{25}$ found out that even minor creatinine increases ( $\Delta$ creatinine $25 \%$ to $49 \%$ above baseline but $<0.3 \mathrm{mg} / \mathrm{dL}$ ) were associated with a two-fold increased risk of death and 2 days longer stay in hospital. Despite the increase of the cisplatin dosage up to $175 \mathrm{mg} / \mathrm{m}^{2}$ BSA, significantly lower creatinine levels could be obtained by the application of cytoprotection 1 week postoperatively and at discharge in our study.

We also observed that the creatinine increase correlated with the extent of the surgical intervention. Patients who underwent EPP presented noticeably higher serum levels than patients who underwent $P / D$ or $\mathrm{eP} / \mathrm{D}$. Actually, higher levels of serum creatinine were expected particularly after lung-sparing procedures due to resorption via the lung surface. ${ }^{15}$ Therefore, radical resection (EPP) might also play an important role in postoperative renal function.

The real intrathoracic concentration of cisplatin depends on various factors (eg, extent of resection, pleural cavity, BMI). Based on our experience and data, the perfusion volume for HITOC including the chemotherapeutic agents is approximately $4 \mathrm{~L}$ in all cases and therefore the intrathoracic concentration should be at a comparable high level. The increase of the cisplatin dosage induced no higher rate 

creatinine depending on the dosage of cisplatin
FIGURE 1 Perioperative serum

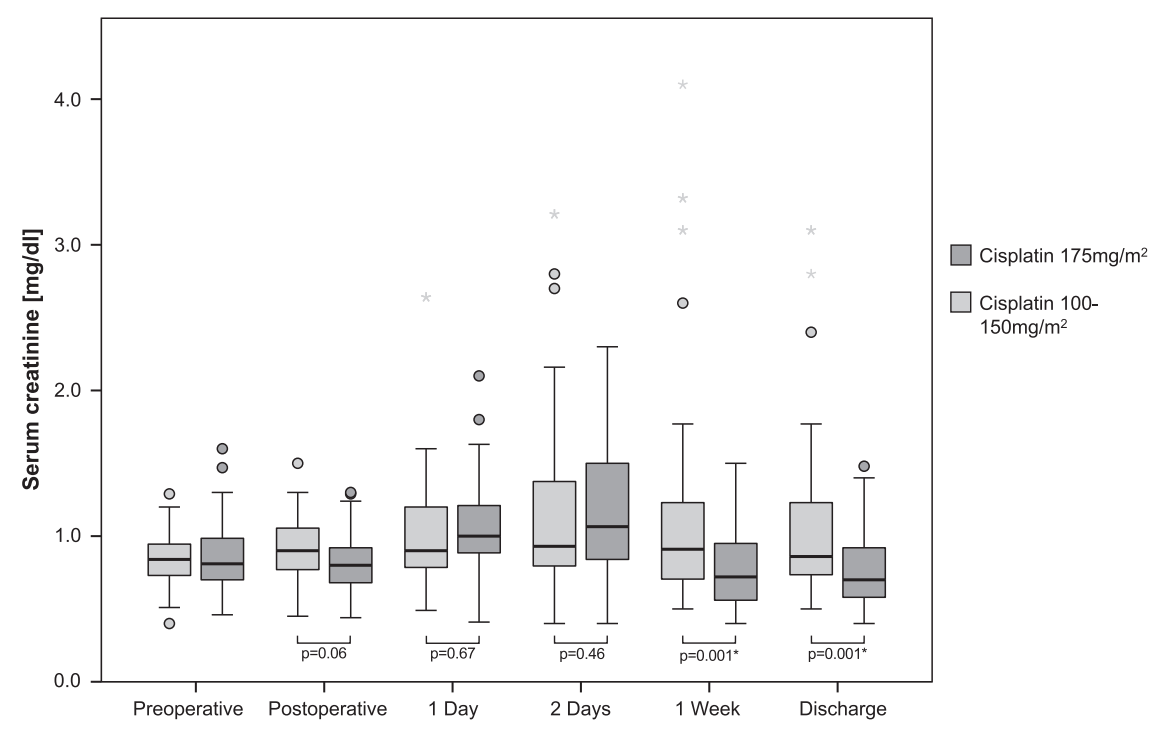

FIGURE 2 Perioperative serum creatinine depending on the application of cytoprotection

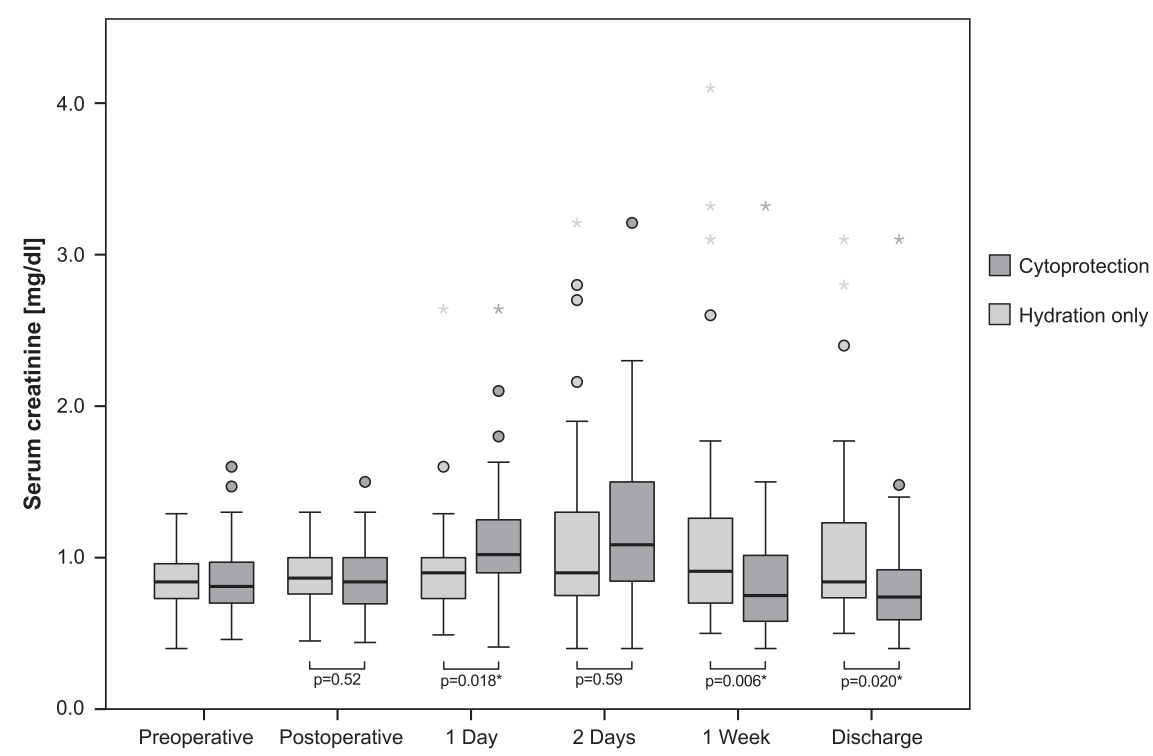

of complications but might provide a potential better local tumor control and overall survival. ${ }^{5,7,10}$

The limitations of the study include the single-center design in selected patients and the retrospective nature. For the last 10 years, we routinely perform HITOC in patients who are scheduled for surgical cytoreduction. Therefore, we can not provide a control group without HITOC. Also, the dose of cisplatin was increased at the same time as cytoprotection was introduced, the effect of cytoprotection on postoperative creatinine and renal insufficiencies in our evaluation was probably attenuated. Furthermore, this was a heterogeneous group of patients with different tumor entities and therapeutical algorithms.

\section{5 | CONCLUSIONS}

In our opinion, cytoprotection is one of the most important tasks in the prevention of postoperative renal complications after HITOC. Aside from perioperative fluid management, amifostine and sodium thiosulfate seem to be effective in protecting the renal function from cisplatin-induced nephrotoxicity. This is expressed in a milder increase in serum creatinine levels after HITOC. By implementing this cytoprotective treatment, higher intracavitary cisplatin doses can be administered without raising the rate of renal insufficiencies. However, special caution is still required in patients with preoperatively elevated creatinine levels and/or known renal insufficiency.

\section{ACKNOWLEDGMENTS}

Open access funding enabled and organized by Projekt DEAL.

\section{CONFLICT OF INTEREST}

The authors declare that there are no conflict of interest.

\section{ORCID}

Till Markowiak (D) http://orcid.org/0000-0002-5364-6206

\section{REFERENCES}

1. Ried $M$, Potzger $T$, Braune $N$, et al. Cytoreductive surgery and hyperthermic intrathoracic chemotherapy perfusion for malignant 
pleural tumours: perioperative management and clinical experience. Eur J Cardiothorac Surg. 2013;43(4):801-807.

2. Sugarbaker DJ, Gill RR, Yeap BY, et al. Hyperthermic intraoperative pleural cisplatin chemotherapy extends interval to recurrence and survival among low-risk patients with malignant pleural mesothelioma undergoing surgical macroscopic complete resection. J Thorac Cardiovasc Surg. 2013;145(4):955-963.

3. Tilleman TR, Richards WG, Zellos L, et al. Extrapleural pneumonectomy followed by intracavitary intraoperative hyperthermic cisplatin with pharmacologic cytoprotection for treatment of malignant pleural mesothelioma: a phase II prospective study. I Thorac Cardiovasc Surg. 2009;138(2):405-411.

4. Yellin A, Simansky DA, Paley $M$, Refaely $Y$. Hyperthermic pleural perfusion with cisplatin: early clinical experience. Cancer. 2001;92(8): 2197-2203.

5. Zellos L, Richards WG, Capalbo L, et al. A phase I study of extrapleural pneumonectomy and intracavitary intraoperative hyperthermic cisplatin with amifostine cytoprotection for malignant pleural mesothelioma. J Thorac Cardiovasc Surg. 2009;137(2):453-458.

6. Madias NE, Harrington JT. Platinum nephrotoxicity. Am J Med. 1978;65(2):307-314.

7. Richards WG, Zellos L, Bueno R, et al. Phase I to II study of pleurectomy/decortication and intraoperative intracavitary hyperthermic cisplatin lavage for mesothelioma. J Clin Oncol. 2006; 24(10):1561-1567.

8. Ishibashi H, Kobayashi M, Takasaki C, Okubo K. Interim results of pleurectomy/decortication and intraoperative intrapleural hyperthermic cisplatin perfusion for patients with malignant pleural mesothelioma intolerable to extrapleural pneumonectomy. Gen Thorac Cardiovasc Surg. 2015;63(7):395-400.

9. McKibbin T, Cheng LL, Kim S, et al. Mannitol to prevent cisplatininduced nephrotoxicity in patients with squamous cell cancer of the head and neck ( $\mathrm{SCCHN}$ ) receiving concurrent therapy. Support Care Cancer. 2016;24(4):1789-1793.

10. Burt BM, Richards WG, Lee HS, et al. A phase I trial of surgical resection and intraoperative hyperthermic cisplatin and gemcitabine for pleural mesothelioma. J Thorac Oncol. 2018;13(9):1400-1409.

11. Weichert-Jacobsen KJ, Bannowski A, Küppers F, Loch T, Stöckle M. Direct amifostine effect on renal tubule cells in rats. Cancer Res. 1999;59(14):3451-3453.

12. Bellomo R, Ronco C, Kellum JA, et al. Acute renal failure - definition, outcome measures, animal models, fluid therapy and information technology needs: the Second International Consensus Conference of the Acute Dialysis Quality Initiative (ADQI) Group. Crit Care. 2004;8(4):R204-R212.

13. Mehta RL, Kellum JA, Shah SV, et al. Acute kidney injury network: report of an initiative to improve outcomes in acute kidney injury. Crit Care. 2007;11(2):R31.

14. Ried M, Potzger T, Braune N, et al. Local and systemic exposure of cisplatin during hyperthermic intrathoracic chemotherapy perfusion after pleurectomy and decortication for treatment of pleural malignancies. J Surg Oncol. 2013;107(7):735-740.

15. Ratto GB, Civalleri D, Esposito $M$, et al. Pleural space perfusion with cisplatin in the multimodality treatment of malignant mesothelioma: a feasibility and pharmacokinetic study. J Thorac Cardiovasc Surg. 1999;117(4):759-765.

16. Ried M, Lehle K, Neu R, et al. Assessment of cisplatin concentration and depth of penetration in human lung tissue after hyperthermic exposure. Eur J Cardiothorac Surg. 2015;47(3):563-566.

17. Kerscher C, Ried M, Hofmann HS, Graf BM, Zausig YA. Anaesthetic management of cytoreductive surgery followed by hyperthermic intrathoracic chemotherapy perfusion. J Cardiothorac Surg. 2014;9:125.

18. Fukushima $\mathrm{K}$, Okada $\mathrm{A}$, Oe $\mathrm{H}$, et al. Pharmacokinetic-pharmacodynamic analysis of cisplatin with hydration and mannitol diuresis: the contribution of urine cisplatin concentration to nephrotoxicity. Eur J Drug Metab Pharmacokinet. 2018;43(2):193-203.

19. Kemp G, Rose P, Lurain J, et al. Amifostine pretreatment for protection against cyclophosphamide-induced and cisplatin-induced toxicities: results of a randomized control trial in patients with advanced ovarian cancer. J Clin Oncol. 1996;14(7):2101-2112.

20. Kouvaris JR, Kouloulias VE, Vlahos LJ. Amifostine: the first selectivetarget and broad-spectrum radioprotector. Oncologist. 2007;12(6): 738-747.

21. Hensley ML, Hagerty KL, Kewalramani T, et al. American Society of Clinical Oncology 2008 clinical practice guideline update: use of chemotherapy and radiation therapy protectants. J Clin Oncol. 2009;27(1):127-145.

22. Markman M, Cleary S, Howell SB. Nephrotoxicity of high-dose intracavitary cisplatin with intravenous thiosulfate protection. Eur J Cancer Clin Oncol. 1985;21(9):1015-1018.

23. Howell SB, Taetle R. Effect of sodium thiosulfate on cis-dichlorodiammineplatinum(II) toxicity and antitumor activity in L1210 leukemia. Cancer Treat Rep. 1980;64(4-5):611-616.

24. Richards WG, Zellos L, Bueno R, Hartigan P, Sugarbaker DJ. 178 Sequential amifostine and sodium thiosulfate improve renal protection during intracavitary high-dose cisplatin lavage. Lung Cancer. 2006;54:S43.

25. Kork F, Balzer F, Spies CD, et al. Minor postoperative increases of creatinine are associated with higher mortality and longer hospital length of stay in surgical patients. Anesthesiology. 2015;123(6):1301-1311.

How to cite this article: Markowiak T, Kerner N, Neu R, et al. Adequate nephroprotection reduces renal complications after hyperthermic intrathoracic chemotherapy. J Surg Oncol. 2019;120:1220-1226. https://doi.org/10.1002/jso.25726 